\title{
Changes in nutritional status after minimally invasive oesophagectomy with an enhanced recovery after surgery (ERAS) programme \& aggressive nutritional intervention; Results of a prospective investigation
}

\author{
E. Ni Bhuachalla ${ }^{1}$, S. Cushen ${ }^{1}$, T. Murphy ${ }^{2}$ and A. Ryan ${ }^{1}$ \\ ${ }^{1}$ School of Food \& Nutritional Sciences, University College Cork, Cork, Republic of Ireland and ${ }^{2}$ Department of \\ Surgery, Mercy University Hospital, Cork, Ireland
}

Oesophagectomy represents an exemplar of controlled major trauma, with marked metabolic, immunologic, and physiologic changes as well as an associated high incidence of complications. Nutritional status can be impaired post-operatively due to the hypermetabolic response to surgery and eating related symptoms such as anorexia, early satiety and acid reflux. Early aggressive nutrition support may limit catabolism but its role in an enhanced recovery after surgery (ERAS) protocol after major upper gastrointestinal surgery is unclear.

The aim of this study was to examine the nutritional outcomes in a cohort of patients presenting for minimally invasive oesophagectomy (MIO) at a specialist centre from March 2012 to March 2015. All patients had histologically proven adenocarcinoma or squamous cell carcinoma of the oesophagus and were enrolled in a standardised multidisciplinary ERAS protocol including written patient education with daily treatment targets, pre-emptive analgesia, early structured mobilisation and early enteral feeding via a needle catheter jejunostomy tube which commenced on post-op day 1 . Oral diet was introduced early and all patients were discharged on overnight enteral feeding for 1 month post-op. Nutritional outcomes (including body composition assessment by both computed tomography $(\mathrm{CT})^{(1)}$ and multi-frequency bioelectrical impedance analysis (BIA)), progression to oral diet and patient-reported global quality of life (QOL) scores (EORTC QLQ-C30) were prospectively collected at baseline, and at 1, 3 and 6 months post-op.

In total 54 patients gave signed informed consent. The mean age was 62 years (SD 9). There were 40 males (74 \%) and 14 females (26\%). The mean percentage weight loss pre-op was $4 \cdot 7 \%$ (SD 5.3). The mean pre-op BMI was in the overweight category $(28 \cdot 6 \mathrm{~kg} /$ $\mathrm{m}^{2}$, SD 5.3), [range 16.9-43.3]. Only $15 \%$ of patients had a BMI in the normal range, $29(54 \%)$ were overweight and $16(29 \%)$ were obese. Based on CT assessment of body composition $47 \%(n=22)$ met the criteria for sarcopenia pre-op ${ }^{(1)}$. Oral diet was introduced after a mean of 4.7 days (SD 2.7) and jejunostomy feeds were switched to night time only. The mean length of stay (LOS) was 11 days (SD 13). Sarcopenic patients spent on average 5.2 days longer in hospital than non-sarcopenic (14 days (SD 19) Vs 8.8 days (SD 3.6), $\mathrm{p}=\mathrm{ns}$ ). On discharge $73 \%$ were tolerating texture B (minced/mashed) half portions and $27 \%$ were tolerating texture $\mathrm{C}$ (puree) half portions.

Weight loss was very frequent following MIO with weight decreasing from $83 \mathrm{~kg}(\mathrm{SD} 15)$ pre-op to $79 \cdot 9$ (SD 12.6) at $1 \mathrm{month}(\mathrm{p}=$ 0.0001 ) and $74.2 \mathrm{~kg}\left(\mathrm{SD} 11.5\right.$ ) by 6 months post-op. BMI dropped significantly from $28.8 \mathrm{~kg} / \mathrm{m}^{2}$ (SD 5.4 ) $\mathrm{pre}-\mathrm{op}$ to $25.5 \mathrm{~kg} / \mathrm{m}^{2}$ (SD 3.4 ) at 6 months post-op $(\mathrm{p}=0.0001)$. Based on BIA assessment of body composition the mean fat mass $(\mathrm{kg})$ decreased significantly postop from $24.1 \mathrm{~kg}(\mathrm{SD} 8.3)$ pre-op to $22.8 \mathrm{~kg}(\mathrm{SD} 8.1)$ by 6 months $(\mathrm{p}=0.03)$. Lean tissue mass decreased from $48.2 \mathrm{~kg}(\mathrm{SD} 12)$ to 42.2 $\mathrm{kg}(\mathrm{SD} 9.5) \mathrm{p}=0.016$. There was no impact on sarcopenia on global QOL scores at 1, 3 or 6 months post-op. Global QOL decreased at 1 month post-op but returned to baseline by 3 months post-op.

In conclusion: an ERAS protocol which includes early aggressive nutrition support and introduction of oral diet post MIO is associated with early discharge from hospital and return of quality of life scores by 3 months post op. There is a significant decline in nutritional status and in particular lean body mass, however this seems to stabilise at 3 months post op at which time QOL scores return to baseline. This feeding protocol (early introduction of oral diet, jejunostomy feeding and discharge on night jejunostomy feeding) is well tolerated, attenuates weight loss and assists in the post-operative recovery.

1. Mourtzakis M, Prado CM, Lieffers JR, Reuman T, McCargar LJ, Baracos VE (2008) A practical and precise approach to quantification of body composition in cancer patients using computed tomography images acquired during routine care. Appl Physiol Nutr Metab 33, 997-1006. 